\title{
Atrial fibrillation before 50 years: epidemiological, clinical and etiological aspects in Guinean cardiology
}

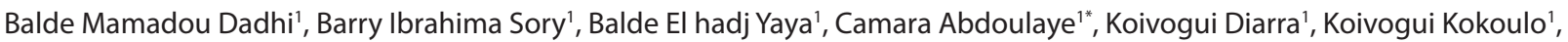 \\ Samoura Aly' ', Doumbouya Mohamed', Barry Mamadou Alpha', Soumaro Morlaye', Sylla Djibril², Beavogui Mariama' and \\ Conde Mamady ${ }^{1}$
}

*Correspondence: mariboudou@gmail.com

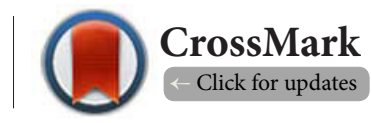

${ }^{1}$ Service of Cardiology Ignace Deen CHU Conakry Republic of Guinea.

${ }^{2}$ Medico-surgical emergency services of Donka National Hospital Republic of Guinea.

\begin{abstract}
Atrial fibrillation is the most common heart rhythm disorder in humans. Al though it mainly affects the elderly, it can also be present in young people. In the absence of treatment, atrial fibrillation can have serious complications, including stroke or heart failure. The absence of an earlier study on AF before 50 years in Guinea, the multiplicity of etiologies motivated the present work with the objectives of determining the magnitude of AF before 50 years and identify the most common etiology. This was a prospective study that included 20 cases of atrial fibrillation in subjects under 50 years of age in the cardiac department of Ignace Deen National Hospital between 1st December 2017 and 30th June 2018. Included in this study were all patients under the age of 50, regardless of sex, who have an FA diagnosed on a resting electrocardiogram. During the study period 80 cases of FA were collected on 398 hospitalizations or a hospital frequency of $20.1 \%$ of which 20 cas of FA in the subjects aged under 50 years were identified either a frequency of $5.02 \%$. The most frequent age group was 20 to 29 years old, ie $40 \%$ with the extreme age of 11 and 49 for a median age of 34.2 years. The female gender was predominant, $65 \%$ versus $35 \%$ of the male gender. Valvulopathies and pericarditis accounted for the most common etiologies $(60 \%$ and $15 \%$, respectively). Atrial fibrillation is not only the prerogative of the elderly. Before the age of 50, the etiology is dominated by valvulopathies because of the endemicity of rheumatic fever in our tropical environments.
\end{abstract}

Keywords: Atrial fibrillation, less than 50 years.epidemiology, clinical, etiology, guinea

\section{Introduction}

Atrial fibrillation (AF) is the most common heart rhythm disorder in humans. Al though it mainly affects the elderly, it can also be present in young people. In the absence of treatment, atrial fibrillation can have serious complications, including stroke or heart failure. Atrial fibrillation causes a wide range of more or less marked symptoms [1]. The severity of AF is related to the risk of thromboembolism (TE) and the risk of heart failure which is a function of the underlying cardiac disease [2]. It can be suspected clinically but can only be characterized on the ECG [2]. AF affects $3 \%$ of patients over 45 and $6.5 \%$ over 65 years. It is associated with a doubled mortality risk, an increase in strokes (risk multiplied on average by 4.5 times) and a risk of heart failure of 2 to 3 times higher [3].
Atrial fibrillation is responsible for 15 to $20 \%$ of all strokes [3]. One of the first explanations that come to mind when trying to elucidate the reason for the increase in the number of detected cases of FA In fact, it results from the frequent discretion of the symptoms of this arrhythmia [4]. It is observed, in fact, that FA produces typical symptoms only in 2/3 of the cases; in others, it remains clinically silent, or is accompanied by atypical and unclassifiable symptoms [5]. It can easily go unnoticed. Nowadays, more frequent use of the electrocardiogram, from primary care or screening examinations, has made it possible to identify numerous cases that previously would have been ignored [5]. Dysrhythmia is relatively easy but its therapeutic management is complex. The etiologies of FA are dominated in our context by valvular causes due to the endemic rheumatic 
fever [6]. Management includes the search for underlying heart disease or curable cause, but also aims to: Prevent thromboembolic risk, restore and maintain sinus rhythm. If the latter option is not possible or indicated, control of ventricular rate to suppress symptoms and limit hemodynamic repercussions. Its management tends to be codified in recent years with the publication of several scientific works in the medical literature. This consensual approach even led to the homogenization of European and American recommendations within the same guidelines in 2006 [6]. The absence of an earlier study on AF before 50 years in Guinea, the multiplicity of etiologies motivated the present work with the objectives of determining the extent of AF before 50 years of age and identifying the most common etiology.

\section{Patients and methods}

This is a prospective study that examined 20 cases of atrial fibrillation in subjects aged less than 50 years in the cardiology department of Ignace Deen National Hospital between 1st December 2017 and 30th June 2018. This study included all patients under the age of 50 , regardless of sex, who have an FA diagnosed on a resting electrocardiogram. The following parameters were analyzed in all patients included: Age, sex, occupation, functional signs, cardiovascular risk factors, etiology of FA, complications, outcomes of electrocardiogram, trans-thoracic ultrasound and biological assessment and treatment. Data capture and analysis were done using the MS word 2007, Excell 2007 and epi software info 3.7.4.

\section{Results}

\section{Frequency}

Eighty-five cases of AF were recorded in 398 hospitalizations, ie a hospital frequency of $20.1 \%$ of which 20 cases of FA in subjects aged under 50 years were identified, ie a frequency of 5.02\% (Tables 1-11 and Figure 1).

\section{Discussion}

During the study period, 85 cases of FA were recorded in 398 hospitalizations, ie a hospital frequency of $20.1 \%$ of which 20 cases of FA in subjects under 50 years of age were identified at a frequency of $5.02 \%$. Our result is close to those reported by M'BAYE A. et al in Senegal in 2010 of $5.35 \%$ and Coulibaly

Table 1. Distribution by age group.

\begin{tabular}{lll}
\hline Age groups & Effective & $\%$ \\
\hline $10-19$ & 4 & 20 \\
$\mathbf{2 0 - 2 9}$ & $\mathbf{8}$ & $\mathbf{4 0}$ \\
$30-39$ & 5 & 25 \\
$40-49$ & 3 & 15 \\
Total & 20 & 100,00 \\
\hline
\end{tabular}

Extreme ages of 11 and 49 years; Median age of 34.2 years

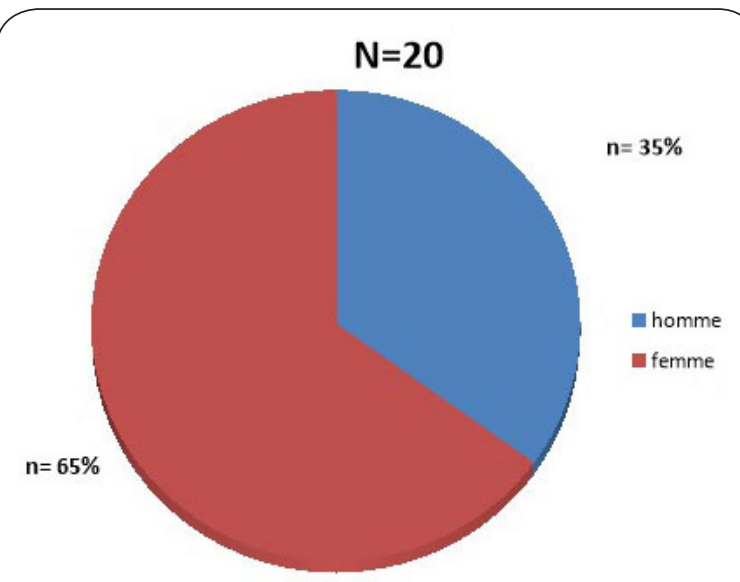

Figure 1. Distribution by gender.

Table 2. Distribution of patients by socio-occupational categories.

\begin{tabular}{lll}
\hline Socioprofessional categories & Effective & $\%$ \\
\hline Household & 10 & 50 \\
Official & 1 & 5 \\
Worker & 3 & 15 \\
Pupil/Student & 6 & 30 \\
Total & 20 & 100,00 \\
\hline
\end{tabular}

Table 3. Distribution of patients by clinical signs.

\begin{tabular}{lll}
\hline Clinical Signs & Effective & \% \\
\hline Dyspnea & 16 & 80 \\
Palpitations & 20 & 100 \\
Chest pain & 10 & 50 \\
Cough & 6 & 30 \\
\hline
\end{tabular}

Table 4. Distribution of patients by etiologies.

\begin{tabular}{lll}
\hline Etiologies & Effective & \% \\
\hline pericarditis & 3 & 15 \\
HTA & 2 & 10 \\
Heart Valve & 12 & 60 \\
$\begin{array}{l}\text { Congenital heart } \\
\text { disease }\end{array}$ & 1 & 5 \\
hyperthyroidism & 2 & 10 \\
Total & 20 & 100,00 \\
\hline
\end{tabular}

HTA: Hypertension 
Table 5. Distribution of patients by electrical signs.

\begin{tabular}{lll}
\hline Other electrical signs & Effective & \% \\
\hline LVH & 17 & 85 \\
HAG & 11 & 55 \\
HVD & 8 & 40 \\
HAD & 4 & 20 \\
ESV & 2 & 10 \\
Flutter & 2 & 10 \\
\hline
\end{tabular}

LVH: hypertrophic left ventricle

HAG: left atrial hypertrophy

HVD: right ventricular hypertrophy

HAD: right atrial hypertrophy

ESV: ventricular extrasystoles

Table 6. Distribution of patients by complications.

\begin{tabular}{lll}
\hline Complications & Effective & \% \\
\hline Stroke & 2 & 10 \\
Heart failure & 16 & 80 \\
Haemorrhage related to AVK & 1 & 5 \\
\hline
\end{tabular}

AVK: Anti vitamin K

Table 7. Distribution of patients according to ultrasound anomalies.

\begin{tabular}{lll}
\hline Ultrasound abnormalities & Effective & $\%$ \\
\hline Dilation of OG & 17 & 85 \\
Dilation of LV & 14 & 70 \\
Dilation straight cavities & 12 & 60 \\
Valvular lesions & 12 & 60 \\
Altered ejection fraction & 16 & 80 \\
Pericardial effusion & 3 & 15 \\
CIV & 1 & 5 \\
\hline
\end{tabular}

OG: left atria, LV: left ventricle, CIV: inter ventricular communication

Table 8. Distribution of patients by ejection fraction.

\begin{tabular}{lll}
\hline Ejection fraction & Effective & \% \\
\hline Normal ejection fraction & 4 & 20 \\
Altered ejection fraction & 16 & 80 \\
Total & 20 & 100 \\
\hline
\end{tabular}

Table 9. Distribution of patients according to valvular lesions.

\begin{tabular}{lll}
\hline Valvular lesions & Effective & \% \\
\hline Mitral insufficiency & 5 & 41,66 \\
Mitral disease & 3 & 25 \\
Aortic insufficiency & 2 & 16,66 \\
PVMAo & 2 & 16,66 \\
Total & 12 & 100 \\
\hline PVMAo: poly mitral and aortic valvulopathy
\end{tabular}

Table 10. Distribution of patients according to biological examinations.

\begin{tabular}{lll}
\hline Biological examinations & Effective & \% \\
\hline Creatinine & 12 & 60 \\
High urea & 5 & 25 \\
Normal blood sugar & 20 & 100 \\
$<1.26 \mathrm{~g} / \mathrm{l}$ & & \\
$\mathrm{TSH}>0,27 \mathrm{UI} / 1$ & 2 & 10 \\
\hline
\end{tabular}

TSH : Thyroid stimulating hormon

Table 11. Distribution of patients by treatment received.

\begin{tabular}{lll}
\hline Treatment received & Effective & \% \\
\hline Beta-blockers & 16 & 80 \\
digitalis & 8 & 40 \\
Amiodarone & 18 & 90 \\
AVK & 17 & 85 \\
AAP & 3 & 15 \\
IEC & 16 & 80 \\
Anti aldostérone & 16 & 80 \\
\hline
\end{tabular}

AAP: Anti aggregating platelet

IEC: Converting enzyme inhibitor

I. et al in Cote d'Ivoire in 2010 of 5.5\% [7,8]. difference is that they took any age confused and in our series the duration of study was 7 months.

The most frequent age group was 20 to 29 years old, ie $40 \%$ with the extreme age of 11 and 49 for a median age of 34.2 years. The female gender was predominant, $65 \%$ versus $35 \%$ of the male gender. Our results are in line with those found in the work of SACCA-VEHOUNKPE J. et al in Benin in 2014 and M'BAYE A. et al. in Senegal in 2010 which respectively report a female predominance of $50.6 \%$ compared to $49.4 \%$ for men [9] and $68.6 \%$ of women against $13.3 \%$ of men [7] unlike that of COULIBALY I. et al in Cote d'Ivoire in 2010, who report a male 
Abdoulaye et al. Cardiovascular System 2020,

predominance of $52 \%$ versus $48 \%$ of women with a sex ratio of 1.08 [8]. The housewives were the most represented socioprofessional layer, ie $50 \%$ followed by pupils / students, ie $30 \%$. Many studies in a tropical environment show the predominance of these two socio-professional layers. This is explained by the fact that these layers are the most disadvantaged in our environment and the cost are exposed to all kinds of diseases including cardiovascular. The clinical signs presented by the patients were respectively: $100 \%$ palpitations, $80 \%$ dyspnea, $50 \%$ chest pain and $30 \%$ cough, all of which are nonspecific signs of heart failure, in which more than half of the patients are hospitalized. Valvulopathies and pericarditis accounted for the most frequent etiologies ( $60 \%$ and $15 \%$ respectively) .M'BAYE A. et al. in Senegal in 2010 [7] and COULIBALY S. et al in Mali in 2012 [10] report the predominance of non-valvular causes of FA including hypertension and coronary heart disease contrary to our results or valvulopathies predominate. This predominance in our series is justified by the fact that valvular lesions caused by rheumatic fever are still prevalent in the tropics and represent one of the leading causes of illness in children and young adults on the one hand and others in children. the series of M'BAYE A. et al. in Senegal in 2010 [7] and COULIBALY S. et al in Mali [10] included subjects of all ages. Left ventricular hypertrophy was associated with $A F$ in $85 \%$ of patients. This result is superior to those of COULIBALY S. et al in Mali in 2012 [10] and Niankara [13] who reported respectively $13.3 \%$ and $50 \%$ of ventricular hypertrophy associated with FA.

The complications encountered were $80 \%, 10 \%$ and $5 \%$, respectively, of heart failure, stroke and hemorrhagic stroke. Hemodynamically, the loss of atrial contraction and the poorly controlled ventricular rate lead to a reduction in blood pressure. cardiac output and increased pulmonary capillary pressure which may cause or decompensate heart failure [11]. Chronic fast AF may also result in left ventricular dysfunction achieving a generally reversible cardiac failure picture after ventricular rate control. The loss of atrial contractility is associated with the formation of intra-atrial thrombi which can induce systemic embolization [11]. According to the Framingham study, $\mathrm{AF}$ is associated with an increase in mortality of 1.5 to $1.9 \%$, mainly related to thromboembolic complications3 [12]. The annual incidence of a patient's stroke in FA is estimated at $5 \%$, ie 2 to 7 times higher than in the normal population [11]. GU dilatation was the most represented ultrasound anomaly (85\%). Our result is close to that of COULIBALY S. et al in Mali in 2012 [10] or $80 \%$. And is consistent with the literature which states that all circumstances that may lead to stretching or dilation of the left atrium and pulmonary veins are favorable conditions for the development of AF [14]. In order to reduce FA Amiodarone has been prescribed in $90 \%$. To slow down the FA beta blockers and Digitals were used in $80 \%$ and $40 \%$ of cases respectively. For the purpose of preventing thromboembolic events $15 \%$ of patients received PAAs and $85 \%$ received AVK. Inhibitors of the conversion enzyme and anti-aldosterone have been prescribed in the heart failure card, $80 \%$ of cases concomitantly.

\section{Conclusion}

Atrial fibrillation is not only the prerogative of the elderly. Before the age of 50 , the etiology is dominated by valvulopathies and this is due to the endemicity of rheumatic fever in our tropical environments. Heart failure and thromboembolic events are the most common complications.

The lack of trans oesophageal ultrasound, the absence of electrical cardioversion and the classification of FA according to the presence or absence of valvulopathy were the difficulties and limitations of our study.

\section{Competing interests}

The authors declare that they have no competing interests.

Authors' contributions

\begin{tabular}{|l|c|c|c|c|c|c|c|c|c|c|c|c|c|}
\hline Authors' contributions & BMD & BIS & BEY & CA & KD & KK & SA & DM & BMA & SM & SD & BM & CM \\
\hline Research concept and design & $\checkmark$ & $\checkmark$ & $\checkmark$ & $\checkmark$ & $\checkmark$ & $\checkmark$ & $\checkmark$ & $\checkmark$ & $\checkmark$ & $\checkmark$ & $\checkmark$ & $\checkmark$ & $\checkmark$ \\
\hline Collection and/or assembly of data & $\checkmark$ & $\checkmark$ & $\checkmark$ & $\checkmark$ & $\checkmark$ & $\checkmark$ & $\checkmark$ & $\checkmark$ & $\checkmark$ & $\checkmark$ & $\checkmark$ & $\checkmark$ & $\checkmark$ \\
\hline Data analysis and interpretation & $\checkmark$ & $\checkmark$ & $\checkmark$ & $\checkmark$ & $\checkmark$ & $\checkmark$ & $\checkmark$ & $\checkmark$ & $\checkmark$ & $\checkmark$ & $\checkmark$ & $\checkmark$ & $\checkmark$ \\
\hline Writing the article & $\checkmark$ & $\checkmark$ & $\checkmark$ & $\checkmark$ & $\checkmark$ & $\checkmark$ & $\checkmark$ & $\checkmark$ & $\checkmark$ & $\checkmark$ & $\checkmark$ & $\checkmark$ & $\checkmark$ \\
\hline Critical revision of the article & $\checkmark$ & $\checkmark$ & $\checkmark$ & $\checkmark$ & $\checkmark$ & $\checkmark$ & $\checkmark$ & $\checkmark$ & $\checkmark$ & $\checkmark$ & $\checkmark$ & $\checkmark$ & $\checkmark$ \\
\hline Final approval of article & $\checkmark$ & $\checkmark$ & $\checkmark$ & $\checkmark$ & $\checkmark$ & $\checkmark$ & $\checkmark$ & $\checkmark$ & $\checkmark$ & $\checkmark$ & $\checkmark$ & $\checkmark$ & $\checkmark$ \\
\hline Statistical analysis & $\checkmark$ & $\checkmark$ & $\checkmark$ & $\checkmark$ & $\checkmark$ & $\checkmark$ & $\checkmark$ & $\checkmark$ & $\checkmark$ & $\checkmark$ & $\checkmark$ & $\checkmark$ & $\checkmark$ \\
\hline
\end{tabular}

Publication history

Editor: Shiwei Duan, Ningbo University, China.

Received: 26-Mar-2020 Final Revised: 30-July-2020

Accepted: 17-Aug-2020 Published: 31-Aug-2020 


\section{References}

1. Swiss Heart Foundation: active against heart disease and stroke, atrial fibrillation, April 2016.

2. High Authority of Health: Care Pathways / Chronic Disease Service and Support Systems for Atrial Fibrillation February 2014.

3. Allan C. Skanes, MD, FRCPC, Jeff S. Healey, MD, MSc, FRCPC, John A. Cairns, MD, FRCPC et al. Focused 2012 Update of the Canadian Cardiovascular Society Atrial Fibrillation Guidelines. Canadian Journal of Cardiology. 2012; 28:125-136.

4. Frykman V, Frick M, Jensen-Urstad M, et al. Asymptomatic versus symptomatic persistent atrial fibrillation. J Intern Med. 2001; 250:390-397.

5. H. Kulbertus, P. Lancellotti. Atrial fibrillation; an epidemic of old age? Rev Med Liège. 2014; 69:5-6: 301-308.

6. A. Saloua. Atrial fibrillation in the cardiology department of Hassan II University Hospital in Fez: epidemiological and therapeutic features. About 494 cases. Medical Thesis: Fès, 2006; P 2.

7. M'BAYE A. et al. Atrial fibrillation, frequency, etiologic factors, evolution and treatment in a cardiology department in Dakar, Senegal Pan. Afr Med J. 2010.

8. Coulibaly I, Anzouan-Kacou JB, Kouao Konin C, Kouadio SC, Abouo-N'Dori R. Atrial Fibrillation: Epidemiology at the Heart Institute of Abidjan (Côte d'Ivoire). Med Trop. 2010; 70:371-374.

9. SACCA-VEHOUNKPE J., TCHABI Y., HOUNTON N., HOUNKPONOU M, ASSANI S., KEKIN E., HOUENASSI M. RISK FACTORS OF ATRIAL FIBRILLATION IN CARDIOLOGY AT CNHU DE COTONOU (2010-2014). Journal de the Society of Clinical Biology of Benin, 2016; 024: 52-55.

10. COULIBALY S. et al, atrial fibrillation in the cardiology department of the $\mathrm{U}-\mathrm{CHU}$ : clinical, etiological factors and natural evolution.

11. P. Nardo, D. Shah, H. Burri, Ph. Meyer; Atrial Fibrillation - HUG - DMCPRU - Primary Care Medicine - 2013.

12. Wolf $P, A b b o t t ~ R$, Kannel W. Atrial fibrillation as an independent risk factor for stroke: The Framingham Study. Stroke. 1999; 22:983-988.

13. Niankara A. Valvular and non-valvular atrial fibrillations in Burkina Faso. comparative study about 58cas collected at $\mathrm{CHU}$ YO. Specialization Thesis in Cardiology: Bamako, USTTB, FMPOS, 2012.

14. Fuster V, Ryden LE, Cannom DS, Crijns HJ, Curtis AB, Ellenbogen KA et al. ACC / AHA / ESC 2006 Guidelines for the Management of Patients with Atrial Fibrillation: A Report of the American College of Cardiology / American Heart Association Task Force on Practice Guidelines and the European Society of Cardiology Committee for Practice Guidelines (Writing Committee to Revise the 2001 Guidelines for the Management of Patients With Atrial Fibrillation): developed in collaboration with the European Heart Rhythm Association and the Heart Rhythm Society. Circulation. 2006; 114:257-354.

\section{Citation:}

Dadhi BM, Sory BI, Yaya BE, Abdoulaye C, Diarra K, Kokoulo K, Aly S, Mohamed D, Alpha BM, Morlaye S, Djibril S, Mariama B and Mamady C. Atrial fibrillation before 50 years: epidemiological, clinical and etiological aspects in Guinean cardiology. Cardio Vasc Syst. 2020; 8:1. http://dx.doi.org/10.7243/2052-4358-8-1 